\title{
Research Square \\ Ketorolac and Predicted Severe Acute Pancreatitis: A Randomized, Controlled Clinical Trial
}

\section{Shaahin Shahbazi}

Ilam University of Medical Sciences

Zahra Vahdat Shariatpanahi ( $D$ nutritiondata@yahoo.com )

Shaheed Beheshti University of Medical Sciences

\section{Erfan Shahbazi}

Shahid Beheshti University of Medical Sciences School of Nutrition and Food Technology

\section{Research article}

Keywords: Inflammation, Enteral nutrition, CRP, APACHE, Feeding

Posted Date: August 27th, 2020

DOI: https://doi.org/10.21203/rs.3.rs-47968/v1

License: (c) (i) This work is licensed under a Creative Commons Attribution 4.0 International License. Read Full License 


\section{Abstract}

Background: We evaluated effect of ketorolac on reducing the severity of acute pancreatitis.

Methods: Fifty six adult patients, with predicted severe acute pancreatitis were randomly divided into two groups. The patients in the study group received intravenous ketorolac, $10 \mathrm{mg}, 3$ times daily from time of enrollment for a maximum of 5 days as needed along with standard medical treatment. Primary outcome measure was the change in the serum level of hs-CRP. Patients were also followed up in terms of hospitalization duration, need for ICU care, development of organ failure, persistent organ failure, pancreatic necrosis, nutritional assessment and mortality. The study was continued to gather clinical follow up information up to 4 months.

Results: Serum level of hs-CRP was significantly lower in the ketorolac group than in the control group on days 3,4 , and 5 . Organ failure, pseudocyst formation, acute necrotic collection and intensive care unit transfer occurred non-significantly more in the control group than in the ketorolac group. Median days of hospitalization were significantly lower in the study group than in the control group. Based on the Log rank test, survival within 4 months was marginally lower in the control group $(P=0.076)$. The time to start feeding was significantly shorter in the study group than in the control group with no need to artificial enteral nutrition in the ketorolac group. Frequency of NPO (not per oral) was significantly lower in the ketorolac group.

Conclusion: The use of ketorolac may improve feeding and clinical outcomes in severe acute pancreatitis.

ClinicalTrials.gov: NCT02885441, Date: August 31, 2016

\section{Background}

Incidence of acute pancreatitis, an inflammatory condition of the pancreas, ranges from 4.9 to 35 per 100,000 population annually, and approximately 15 to $25 \%$ of all patients with acute pancreatitis develop severe acute pancreatitis ${ }^{1}$. A damaged pancreas releases cytokines and activated pancreatic enzymes into the circulation which could damage pancreas and also develop systemic inflammatory response syndrome (SIRS) in the patient ${ }^{2}$. A compensated anti-inflammatory response syndrome balances the SIRS, resulting in recovery. An imbalance between inflammatory and anti-inflammatory responses results in severe organ failure ${ }^{2}$. Research has further shown the early inhibition of proinflammatory cytokine activity with the administration of IL-10, an anti-inflammatory cytokine able to reduce the severity of experimental pancreatitis in animals ${ }^{3}$ and prevent the development of post endoscopic retrograde cholangiopancreatography (ERCP) pancreatitis in humans ${ }^{4}$. With antipyretic, analgesic, and antiinflammatory properties, ketorolac inhibits cyclooxygenase- 1 and 2 enzymes, reducing the formation of prostaglandin precursors. Other mechanisms proposed for ketorolac which contribute to antiinflammatory effects, include inhibiting chemotaxis, altering lymphocyte activity, inhibiting neutrophil 
aggregation/activation, and decreasing proinflammatory cytokine levels ${ }^{5}$. Because the extent of inflammation correlates with the severity of pancreatitis ${ }^{2}$, it seems that the early reduction of inflammation with ketorolac administration can decrease the development of organ failure and outcomes of morbidity in acute pancreatitis. Additionally, delayed feeding due to pain in acute pancreatitis, and unused gastrointestinal tract, disrupt the tight junction of enterocytes and further increases inflammation and risk of organ failure in patients ${ }^{6}$. In the present research, it was hypothesized that the administration of ketorolac could reduce the inflammation of pancreatitis and organ failure, shorten the duration of hospitalization, control pain and food intolerance, promote earlier feeding and shorten duration of hospitalization. To evaluate this hypothesis, a randomized, controlled clinical trial was established to determine these effects in patients with predicted severe acute pancreatitis.

\section{Methods}

In the present randomized, controlled clinical trial, 56 adult patients, with predicted severe acute pancreatitis were recruited between September 2016 and February 2019, from a university hospital. In the beginning of the study, written informed consent was obtained from patients. The study was approved by the Ilam University of Medical Sciences ethics committee and has therefore been performed in accordance with the ethical standards laid down in the 1964 Declaration of Helsinki and its later amendments. The study adheres to CONSORT guidelines.

Exclusion criteria were heart disease, history of coronary artery bypass graft, hypertension, hemorrhagic diathesis, incomplete hemostasis, high risk of bleeding, hypersensitivity to non-steroidal antiinflammatory drugs (NSAID), concurrent use with aspirin, other NSAIDs or pentoxifylline, active or history of peptic ulcer disease, recent or history of GI bleeding or perforation, inflammatory bowel disease, asthma, pregnancy and lactating women, advanced renal disease, elevated/rising creatinine, and severe hepatic impairment or active hepatic disease.

Patients with the predictors of severe acute pancreatitis according to the guidelines of the American College of Gastroenterology ${ }^{7}$ and International Association of Pancreatology/American Pancreatic Association ${ }^{8}$, approached within the first 72 hours of onset, were included in the study. These predictors are: (1) age $>55$ years, (2) obesity (body mass index $>30 \mathrm{~kg} / \mathrm{m}^{2}$ ), (3) altered mental status, (4) comorbid disease, (5), SIRS (Presence of $>2$ of the following criteria: pulse $>90$ beats $/ \mathrm{min}$, respirations $>20 / \mathrm{min}$ or $\mathrm{PaCO}_{2}<32 \mathrm{~mm} \mathrm{Hg}$, temperature $>38$ or $<36{ }^{\circ} \mathrm{C}$, white blood cell count $>12,000$ or $<4,000$ cells $/ \mathrm{mm}^{3}$ or $>$ 10 percent immature neutrophils), (6) hematocrit (HCT) > 44 percent, (7) rising $\mathrm{HCT}$, (8) elevated blood urea nitrogen (BUN), (9) rising BUN and (10) radiologic findings of pleural effusions or pulmonary infiltrates or multiple or extensive extrapancreatic collections. Albeit we did not consider elevated/rising creatinine in inclusion criteria.

Patients were randomly divided into two groups, and randomization was performed according to a computer-generated random number table. A physician who was not a member of research team was unblinded to the treatment assignment, whereas the patients, investigators, and all clinical personnel 
remained blinded to the randomization. The study group received $10 \mathrm{mg}$ intravenous injection of ketorolac three times a day up to 5 days as needed. Along with the study intervention, the patients were managed by standard medical treatment including intravenous fluid and electrolytes, and other supportive therapies for organ failure, as indicated. On admission, high sensitive $\mathrm{C}$ reactive protein (hsCRP) was measured, and standard laboratory tests were conducted. Measurement of hs-CRP was repeated daily up to day 5 of hospitalization.

Following the completion of fluid volume resuscitation (within 24-48 hours of admission), feeding was started for each patient. In patients who could tolerate oral feeding, standard polymeric formula was started at a trophic rate. According to the standards of hospital, in subjects intolerant of oral feeding within 4 days, nasogastric tube was inserted and enteral nutrition with standard polymeric formula was started. Enteral feeding was performed 8 times a day through intermittent technique. The volume of administration was determined based on the calorie needs and tolerance of patients, starting from $50 \mathrm{~mL}$. Calorie needs were measured and calorie intake was recorded daily for each individual during the study period. Primary outcome measure was the change in the serum level of hs-CRP. Patients were also followed up in terms of hospitalization duration, need for ICU care and length of ICU stay, development of organ failure, persistent organ failure, pancreatic necrosis, and mortality. Organ failure was defined by shock (systolic blood pressure $<90 \mathrm{~mm} \mathrm{Hg}$ ), pulmonary insufficiency (Pao2/Fio2 $\leq 300$ ), or renal failure (serum creatinine $\geq 1.9 \mathrm{mg} / \mathrm{dL}$ ). Nutritional assessment were time of beginning and tolerance to nutrition, daily nutritional administration and the frequency that subjects were made NPO (not per oral) because of pain, nausea or vomiting after refeeding.

The study was continued to gather clinical follow up information up to 4 months.

All authors had access to the study data and reviewed and approved the final manuscript.

\section{Statistical analysis}

We did not find a study with similar objectives for calculating the sample size. We conducted a small pilot study in order to get the required estimates and perform a proper sample size calculation. The minimum sample size estimation for each group was 28 at a power $(1-\beta)$ of $80 \%$ and $a=0.05$ for a 2 -arm parallel study to detect a difference of $24 \mathrm{mg} / \mathrm{L}$ in hs-CRP serum level on day 3 , obtained from the pilot study.

Data were analyzed using SPSS software V.21. Characteristics of the patients were expressed in frequencies and percentages for categorical variables. The quantitative variables with normal distribution are summarized as mean and standard deviation. Non-normal quantitative variables are presented by median and their first and third quartiles. Differences between variables were evaluated using t-test, Mann-Whitney U- test, and the Chi-square or Fisher Exact test. Analysis of covariance (ANCOVA) was employed in order to detect the exact effect of ketorolac on the serum level of CRP and the duration of hospitalization. In that analysis, adjustment was done based on the covariates including BMI and Acute Physiologic Assessment and Chronic Health Evaluation (APACHE) II score. The Log rank test was applied for mortality. A two-sided $\mathrm{P}$ value of less than 0.05 was considered significant. 


\section{Results}

Seventy-four patients met all the inclusion criteria and were eligible for consent. Eighteen refused to participate, and 56 patients consented to participation, completed the study, and were randomly divided into two groups of ketorolac and control. Disposition of patients throughout the study is shown in Fig. 1. There were 24 men and 32 women with a mean age of $47.9 \pm 11.5$ (range 26-51). The most frequent cause of severe acute pancreatitis was biliary stones in both groups which were also similar with regards to age, sex, body mass index, APACHE, SIRS score and pancreatic necrosis or organ failure on admission. The basal characteristics of patients are presented in Table 1.

Table 1

Basal characteristics of patients

\begin{tabular}{|llll|}
\hline & $\begin{array}{l}\text { Control group } \\
(\mathbf{n = 2 8})\end{array}$ & Study group(n=28) & P value \\
\hline Age, year (mean \pm SD) & $48.7 \pm 11$ & $47.1 \pm 12.1$ & 0.59 \\
\hline $\begin{array}{l}\text { Sex, } n(\%) \\
\text { Male }\end{array}$ & $13(46 \%)$ & $11(39 \%)$ & 0.39 \\
Female & $15(54 \%)$ & $17(61 \%)$ & \\
Cause of pancreatitis, $n(\%)$ & & & 1 \\
\hline Biliary stone & $26(93)$ & $26(93)$ & 1 \\
\hline Hypertriglyceridemia & $2(7)$ & $2(7)$ & 0.39 \\
\hline BMI, kg/m ${ }^{2}$ (mean \pm SD) & $29.8 \pm 2.1$ & $28.8 \pm 5.4$ & 0.89 \\
\hline APACHE II (mean \pm SD) & $8 \pm 2.1$ & $7.9 \pm 2.04$ & 0.89 \\
\hline SIRS (mean \pm SD) & $4.07 \pm 1.9$ & $4.14 \pm 2$ & 1 \\
\hline Admission necrosis, $n$ (\%) & $0(0)$ & $0(0)$ & 1 \\
\hline Admission Organ failure, $n$ (\%) & $2(7)$ & $2(7)$ & \\
\hline $\begin{array}{l}\text { BMI, body mass index; SIRS, systemic inflammatory response syndrome; APACHE, Acute Physiology } \\
\text { and Chronic Health Evaluation }\end{array}$ & & & \\
\hline
\end{tabular}

\section{Inflammatory Outcomes}

Table 2 shows the changes in serum hs-CRP level during the study period. Basal CRP level was similar in both groups. There was an insignificant increase in the serum CRP level of the control group on day 2 , 
after which time, it had a decreasing slope. In the ketorolac group, serum CRP level had a decreasing trend from day 1 to day 5 .

Table 2

Inflammatory outcomes of the ketorolac and control groups

\begin{tabular}{|c|c|c|c|c|}
\hline & $\begin{array}{l}\text { Study group } \\
(n=28)\end{array}$ & Control group $(n=28)$ & P value* & P value ${ }^{\star \star}$ \\
\hline \multicolumn{5}{|l|}{$\mathrm{CRP}, \mathrm{mg} / \mathrm{L},($ mean $\pm \mathrm{SD})$} \\
\hline Day 1 & $169.7 \pm 24.9$ & $164.9 \pm 25$ & 0.47 & 0.46 \\
\hline Day 2 & $162.9 \pm 31.3$ & $174.6 \pm 20.2$ & 0.10 & 0.13 \\
\hline Day 3 & $129.5 \pm 28.8$ & $157.2 \pm 29.7$ & 0.001 & $<0.001$ \\
\hline Day 4 & $104.8 \pm 32.8$ & $152.2 \pm 33$ & $<0.001$ & $<0.001$ \\
\hline Day 5 & $73.1 \pm 20$ & $114.7 \pm 19$ & $<0.001$ & $<0.001$ \\
\hline CRP, mg/L (change day 1 to 3 ) & $-40.2 \pm 35.8$ & $-7.6 \pm 40.3$ & 0.002 & 0.002 \\
\hline $\mathrm{CRP}, \mathrm{mg} / \mathrm{L}$ (change day 1 to 4 ) & $-64.9 \pm 41.5$ & $-12.7 \pm 38.8$ & $<0.001$ & $<0.001$ \\
\hline $\mathrm{CRP}, \mathrm{mg} / \mathrm{L}$ (change day 1 to 5 ) & $-111.6 \pm 30.5$ & $-50.1 \pm 33.7$ & $<0.001$ & $<0.001$ \\
\hline
\end{tabular}

Serum CRP was reduced to $24 \%$ of enrolment levels on day $3,38 \%$ on day 4 and $66 \%$ on day 5 in the ketorolac group, which is a significant decrease as analyzed by paired t test $(\mathrm{P}<0.001$ in all). This reduction was $4 \%(P=0.3), 8 \%(P=0.09)$ and $30 \%(P<0.001)$ on days 3,4 and 5 , respectively in the control group.

There was a significant difference between ketorolac and the controls regarding the reduction in the serum CRP levels on days 3,4 , and 5 . To control the covariates, APACHE score and BMI were entered in the ANCOVA model, but the results did not change $(P<0.001$ in all). The partial Eta Squared values were $0.21,0.38$, and 0.68 on days 3,4 , and 5 respectively. In other words, the effect of ketorolac on reducing the serum level of CRP increased with time.

\section{Feeding Outcomes}

Nutritional outcomes are reported in Table 3. In both oral and enteral nutrition, the formula was polymeric standard ( $15 \%$ protein) started with trophic feeding. The time interval to start feeding was significantly shorter in the ketorolac group compared to the control group. On the third day of admission, $11(40 \%)$ of patients in the ketorolac group and $3(11 \%)$ patients in the control group tolerated oral feeding. On day 4 , 
oral feeding was started for another $11(40 \%)$ patients in the ketorolac group and $11(39 \%)$ patients in the control group. On day 5, all 28 patients in the ketorolac group were on oral feeding. On day 5 , oral feeding was started for another $8(29 \%)$ patients, and enteral nutrition was started for the remaining $6(21 \%)$ patients in the control group.

Table 3

Clinical outcomes of the ketorolac and control groups

\begin{tabular}{|llll|}
\hline & $\begin{array}{l}\text { Study group } \\
(\mathbf{n = 2 8 )}\end{array}$ & Control group(n=28) & P value \\
\hline Days of hospitalization, (mean \pm SD) & $6.71 \pm 1.08$ & $9.10 \pm 1.22$ & $<0.001$ \\
\hline Need for ICU, $\mathrm{n}(\%)$ & $1(4)$ & $4(14)$ & 0.35 \\
\hline Organ failuret, $\mathrm{n}(\%)$ & $1(4)$ & $5(18)$ & 0.19 \\
\hline Acute necrotic collection, $\mathrm{n}(\%)$ & $0(0)$ & $1(4)$ & 1 \\
\hline Pseudocyst, $\mathrm{n}(\%)$ & $1(4)$ & $3(11)$ & 0.61 \\
\hline tNew onset or Persistence organ failure & & \\
\hline
\end{tabular}

Table 4

Nutritional Outcomes of ketorolac and control groups

\begin{tabular}{|llll|}
\hline & $\begin{array}{l}\text { Study } \\
\text { group } \\
(\mathbf{n = 2 8})\end{array}$ & $\begin{array}{l}\text { Control group(n= } \\
\mathbf{2 8})\end{array}$ & P value* \\
\hline Type of feeding, $(\mathrm{n}, \%)$ & & & 0.023 \\
\hline Oral & $28(100)$ & $22(79)$ & \\
\hline Tube feeding & $0(0)$ & $6(21)$ & 0.001 \\
\hline $\begin{array}{l}\text { Time interval to start feeding(day), (median, Q1- } \\
\text { Q2) }\end{array}$ & $4(3-4)$ & $4(4-5)$ & 0.029 \\
\hline Frequency of NPO, (n, \%) & $3(11)$ & $11(39)$ & $<0.001$ \\
\hline $\begin{array}{l}\text { Delivery to goal calorie (day), } \\
\text { (mean } \pm \text { SD) }\end{array}$ & $6.46 \pm 0.69$ & $8.71 \pm 1.08$ & \\
\hline
\end{tabular}

The ability to deliver nutrition was better in the ketorolac group compared to the control group. The ketorolac group reached the targeted energy intake significantly sooner than the control group. Tolerance to diet (abdominal pain, nausea and vomiting after refeeding) which leads to feeding stoppage was significantly fewer in the ketorolac group. 


\section{Clinical Outcomes}

In the ketorolac group, persistence and new onset organ failure was insignificantly lower (1 case versus 5 cases) compared to the control group. Acute necrotic collection was observed in one patient in the control group during hospitalization. Duration of hospitalization was significantly more in the control group compared to the ketorolac group and the result did not change using ANCOVA model with controlled covariates, APACHE score and BMI $(\mathrm{P}<0.001)$. Following discharge, 1 patient in the ketorolac group and 3 patients in the control group returned to the hospital due to pseudocyst formation.

There was no in hospital mortality in both group. After discharge, four-month mortality was seen in 3 patients of the control group. Based on the Log rank test, survival within 4 months was marginally lower in the control group compared with the ketorolac group, a difference which was not statistically significant $(P=0.076)$.

\section{Discussion}

In this study, the efficacy of ketorolac was evaluated in predicted severe acute pancreatitis. In patients who received ketorolac, serum CRP level was reduced faster, and they were sooner discharged from the hospital. Additionally, in the ketorolac group, a significantly better food tolerance control was observed, feeding was started earlier than the controls, and there was no need for artificial enteral nutrition. Organ failure involvement and mortality were lower in the ketorolac group, though not significantly.

In acute pancreatitis, the extent of inflammation correlates with the severity of pancreatitis and the goal of management is to reduce the inflammation. In the pathogenesis of acute pancreatitis, chemoattraction of leukocytes occurs and proinflammatory cytokines (tumor necrosis factor, interleukins 1, 6, and 8), arachidonic acid metabolites (prostaglandins, platelet-activating factor, and leukotrienes), proteolytic and lipolytic enzymes, and reactive oxygen metabolites are released by activated granulocytes /macrophages. These factors induce pancreatic damage ${ }^{9}$.

In liver, CRP rises steadily in relation to the severity of pancreatitis and in response to interleukin-1 and interleukin-6. C-reactive protein, a sensitive and nonspecific marker of inflammation, can predict complication and prognosis in acute pancreatitis.

As a potent NSAID, ketorolac inhibits cyclooxygenase- 1 and 2 enzymes, preventing the prostaglandin precursors production with analgesic and anti-inflammatory properties.

In a double-blinded randomized control trial, Vege et al. found that use of pentoxifillyne, an inhibitor of tumor necrosis factor in patients with predicted severe acute pancreatitis, was associated with fewer ICU admissions and lower duration of hospitalization ${ }^{10}$. In their study, although serum TNF-a, CRP, interleukin-1 and interleukin- 6 levels decreased more in pentoxifillyne group, the reduction was not statistically significant, which may be due to the small sample size (14 in each group). 
Some studies have evaluated the preventive effects of rectal indomethacin or diclofenac in post-ERCP pancreatitis, with a meta-analysis confirming their protective effect ${ }^{11}$. Another meta-analysis showed that rectal indomethacin administration prior to ERCP is effective for the prevention of post-ERCP pancreatitis in high-risk patients ${ }^{12}$. Phospholipase A2 activity is inhibited by rectal NSAIDs in acute pancreatitis, possibly regulating proinflammatory mediators.

On the other hand, early feeding had an impact on attenuating the inflammatory response by itself ${ }^{13}$. The old approach of putting the pancreas at rest in acute pancreatitis and feeding the patient with parenteral nutrition has been changed. In fact, the results of meta-analysis have shown that the use of gastrointestinal tract for feeding, in severe acute pancreatitis, reduces mortality, infectious complications, organ failure, and surgical intervention rate in comparison to parenteral nutrition ${ }^{14-15}$. American society of enteral and parenteral nutrition suggests that in patients intolerable to oral feeding, enteral feeding should be started as a trophic rate ${ }^{16}$. Patients with moderate to severe acute pancreatitis refuse oral feeding due to nausea, vomiting and abdominal pain secondary to gastric stasis and abdominal distention following pancreas inflammation. Food intolerance is also present in early enteral nutrition ${ }^{17}$. With the increase in pancreatic inflammation, the severity of intolerance increases and feeding becomes more problematic ${ }^{7}$. The results of our study showed that all patients in the ketorolac group tolerated oral feeding without the need for enteral nutrition. Furthermore, the time interval for feeding initiation was shorter in the ketorolac group compared to the control group, which may be due to the potent antiinflammatory properties of ketorolac and its analgesic effects. Therefore, the frequency of NPO was lower in patients receiving ketorolac along with fewer days of hospitalization.

In acute pancreatic, intestinal permeability ranges from mild to severe forms. It has been shown that the use of gastrointestinal tract for feeding, maintains intestinal integrity, and reduces the translocation of bacteria in mesenteric lymph nodes and plasma endotoxin levels ${ }^{18}$. Starvation changes the intestinal microbiotia composition and promotes proinflammatory patterns. American society of parenteral and enteral nutrition states that in patients with moderate to severe acute pancreatitis, failure to start enteral nutrition for more than 3-4 days following admission, ensues the risk of worsened nutrition status and development of SIRS, organ failure and infection ${ }^{16}$. Based on this recommendation, we started enteral nutrition on day of 5 in all patients unable to start feeding. In the control group, 39\% of patients were on artificial enteral nutrition. All patients who received ketorolac started their oral feeding prior to day 5 of admission with no need for artificial enteral nutrition.

Reduced inflammation and earlier feeding observed in the ketorolac group may be the reasons for fewer organ failures and ICU stays, preventing the progress of predicted severe acute pancreatitis to severe forms.

\section{Strengths and weaknesses:}


For the first time, we examined the effect of ketorolac injection on feeding and clinical outcomes in predicted severe acute pancreatitis. Ketorolac was not accompanied by any side effects in our patients, and patients were followed up to investigate the survival within four months.

However, the present study had some weaknesses. As there was no study similar to the present, we chose the changes in serum CRP level for primary end-point, and not the clinical outcomes. Therefore, this study was unable to detect the effect of ketorolac on the clinical outcomes. Although the duration of hospitalization was significantly lower in the ketorolac group, lower organ failure and mortality might also become significant with larger sample sizes. Furthermore, a physician was unblinded to the study intervention, although participants, data collectors, outcome adjudicators and data analyst were unaware of the treatment groups.

\section{Conclusions}

Using ketorolac, we can reduce the time of hospitalization, control inflammation and improve feeding outcomes with no need for enteral nutrition.

\section{Abbreviations}

APACHE: Acute Physiologic Assessment and Chronic Health Evaluation

ANCOVA: Analysis of covariance

BUN: Blood urea nitrogen

ERCP: Endoscopic retrograde cholangiopancreatography

hs-CRP: High sensitive $\mathrm{C}$ reactive protein

NSAID: Non-steroidal anti-inflammatory drugs

SIRS: Systemic inflammatory response syndrome

\section{Declarations}

\section{Ethics approval and consent to participate}

The study was approved by the llam University of Medical Sciences ethics committee and has therefore been performed in accordance with the ethical standards laid down in the 1964 Declaration of Helsinki and its later amendments. The study adheres to CONSORT guidelines. In the beginning of the study, written informed consent was obtained from patients.

\section{Consent for publication}


Not applicable.

\section{Availability of data and materials}

The datasets used and analyzed during this study are available from the corresponding author on reasonable request.

\section{Competing interests}

The authors declare that they have no competing interests.

\section{Funding}

None.

\section{Authors' Contributions}

All authors contributed to the study conception and design. Material preparation, data collection and analysis were performed by S.S, Z.V and E.S. The first draft of the manuscript was written by Z.V and all authors commented on previous versions of the manuscript. All authors read and approved the final manuscript.

\section{Acknowledgements}

We convey our gratitude to the llam University of Medical Sciences, Ilam, Iran.

The authors confirm that the manuscript is original research that has not been published and is not under consideration elsewhere.

\section{References}

1. Vege SS, Yadav D, Chari ST. Pancreatitis. In: GI Epidemiology, 1st ed, Talley NJ, Locke GR, Saito YA (Eds), Blackwell Publishing, Malden, MA 2007.

2. Raraty MG, Connor S, Criddle DN, et al. Acute pancreatitis and organ failure: pathophysiology, natural history, and management strategies. Curr Gastroenterol Rep. 2004; 6(2):99-103.

3. Van Laethem JL, Eskinazi R, Louis $\mathrm{H}$, et al. Multisystemic production of interleukin 10 limits the severity of acute pancreatitis in mice. Gut. 1998; 43:408-413

4. Singh P, Lee T, Davidoff S, et al. Efficacy of Interleukin 10 (IL-10) in the prevention of post-ERCP pancreatitis: a meta-analysis. GastrointestEndosc. 2002; 55:AB150.

5. Howard ML, Isaacs AN, Nisly SA. Continuous infusion nonsteroidal anti-inflammatory drugs for perioperative pain management. J Pharm Pract. 2018; 31(1):66-81.

6. Eslamian G, Ardehali S.H, Baghestani A, et al. Effects of early enteral bovine colostrum supplementation on intestinal permeability in critically ill patients: A randomized, doubleblind, 
placebo-controlled study. Nutrition. 60 (2019) 106-111.

7. Tenner S, Baillie J, DeWitt J, et al. American College of Gastroenterology guideline: management of acute pancreatitis. Am J Gastroenterology. 2013; 108:1400.

8. Working Group IAP/APA Acute Pancreatitis Guidelines. IAP/APA evidence-based guidelines for the management of acute pancreatitis. Pancreatology. 2013; 13:e1.

9. Chan YC, Leung PS. Acute pancreatitis: animal models and recent advances in basic research. Pancreas. 2007; 34:1.

10. Vege SS, Atwal T, Bi Y, et al. Pentoxifylline Treatment in Severe Acute Pancreatitis: A Pilot, DoubleBlind, Placebo-Controlled, Randomized Trial. Gastroenterology 2015; 149:318-320.

11. Elmunzer BJ, Waljee AK, Elta GH, et al. A meta-analysis of rectal NSAIDs in the prevention of postERCP pancreatitis. Gut. 2008; 57:1262-1267

12. Wan J, Ren Y, Zhu Z, et al. How to select patients and timing for rectal indomethacin to prevent postERCP pancreatitis: a systematic review and meta-analysis. BMC Gastroenterol. 2017 Mar 15; 17(1):43.

13. Louie B, Noseworthy T, Hailey D, et al. 2004 MacLean-Mueller Prize Enteral or parenteral nutrition for severe pancreatitis: a randomized controlled trial and health technology assessment. Can J Surg. 2005 Aug; 48(4): 298-306.

14. Cao Y, Xu Y, Lu T, et al. Meta-analysis of enteral nutrition versus total parenteral nutrition in patients with severe acute pancreatitis. Ann Nutr Metab. 2008; 53(3-4):268-275

15. Yi F, Ge L, Zhao J, et al. Meta-analysis: total parenteral nutrition versus total enteral nutrition in predicted severe acute pancreatitis. Intern Med. 2012; 51(6):523-530.

16. McClave SA, Taylor BE, Martindale RG, et al. Guidelines for the Provision and Assessment of Nutrition Support Therapy in the Adult Critically III Patient: Society of Critical Care Medicine (SCCM) and American Society for Parenteral and Enteral Nutrition (A.S.P.E.N.). JPEN Journal of parenteral and enteral nutrition 2016; 40 (2):159-211.

17. Sun JK, Li WQ, Ke L, et al. Early Enteral Nutrition Prevents Intra-abdominal Hypertension and Reduces the Severity of Severe Acute Pancreatitis Compared with Delayed Enteral Nutrition: A Prospective Pilot Study. World J Surg. 2013 37:2053-2060

18. Cicalese L, Sahai A, Sileri P, Rastellini C, Subb otin V, Ford H, Lee K. Acute pancreatitis and bacterial translocation. Dig Dis Sci 2001; 46: 1127-1132.

\section{Figures}




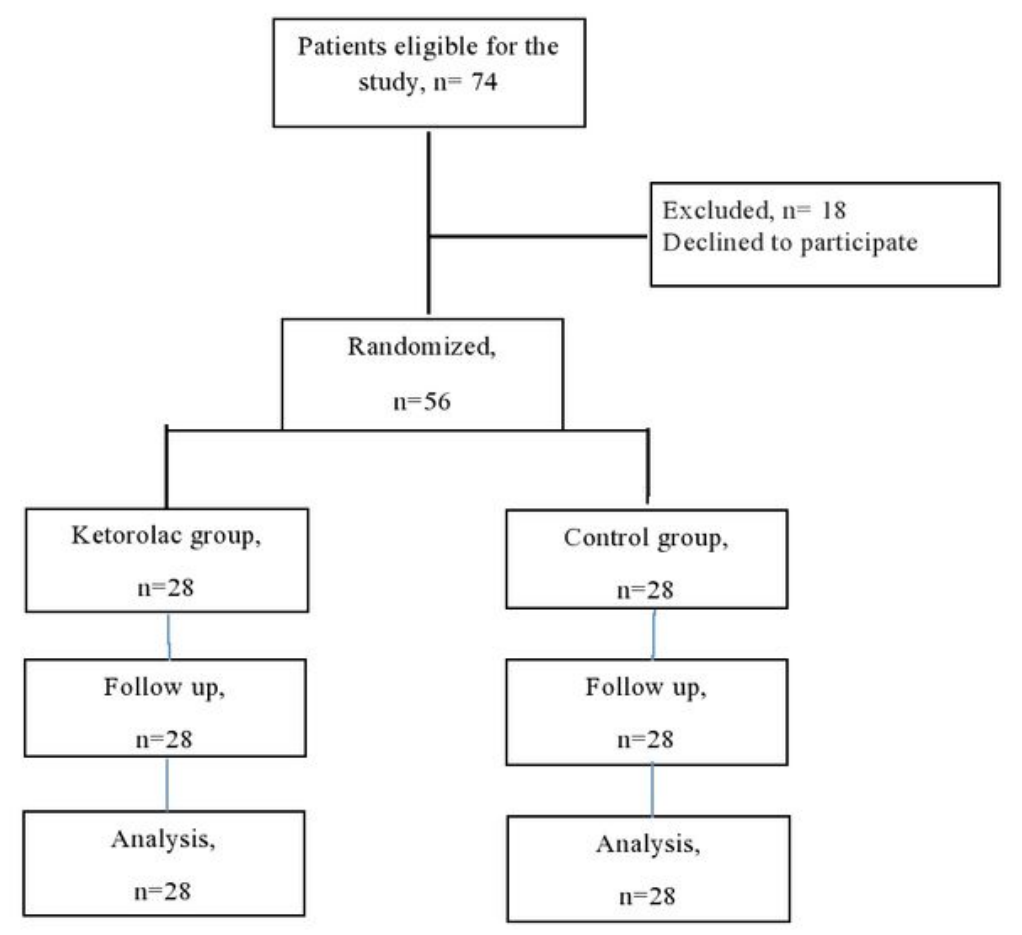

1

\section{Figure 1}

Flowchart of the study participants

\section{Supplementary Files}

This is a list of supplementary files associated with this preprint. Click to download. 
- CONSORT2010Checklist.doc

Page 14/14 